\title{
Presence of high-endothelial venules correlates with a favorable immune microenvironment in oral squamous cell carcinoma
}

\author{
Anna Maria Wirsing ${ }^{1} \cdot$ Ida Korsnes Ervik $^{1} \cdot$ Marit Seppola $^{1} \cdot$ Lars Uhlin-Hansen $^{1,2} \cdot$ Sonja Eriksson Steigen ${ }^{1,2} \cdot$ \\ Elin Hadler-Olsen ${ }^{1,2}$
}

Received: 9 October 2017 / Revised: 20 December 2017 / Accepted: 21 December 2017 / Published online: 7 February 2018

(c) United States \& Canadian Academy of Pathology 2018

\begin{abstract}
Oral squamous cell carcinomas are associated with a poor prognosis, which may be partly due to functional impairment of the immune response. Lymphocyte recruitment to the tumor site is facilitated by high-endothelial venules, whereas expression of programmed-death ligand 1 (PD-L1) can impair T-cell function. Thus, we hypothesize that these factors are important in shaping the immune response in oral squamous cell carcinoma. In the present study, we characterized the immune infiltrate in formalin-fixed, paraffin-embedded tumor samples from 75 oral squamous cell carcinoma patients. We used immunohistochemistry to determine the distribution of immune cell subsets, high-endothelial venules, and PD-L1, as well as quantitative real-time polymerase chain reaction to assess the expression of inflammatory cytokines and chemokines associated with lymphocyte trafficking. Finally, we calculated correlations between the presence of immune cell subsets, the gene expression patterns, high-endothelial venules, PD-L1, and the clinicopathological parameters, including patient survival. The presence of high-endothelial venules correlated with increased number of CD3 $+\mathrm{T}$ cells and CD20 $+\mathrm{B}$ cells, higher levels of the chemokines CXCL12 and CCL21, and lower levels of CCL20, irrespective of the tumors' T stage. In univariate analysis, high levels of CD20 + B cells and CD68+ macrophages, positive high-endothelial venule status, and low $\mathrm{T}$ and $\mathrm{N}$ stages predicted longer patient survival. However, only the presence of high-endothelial venules and a low $\mathrm{T}$ stage were independent positive prognosticators. This indicates that high-endothelial venules are important mediators and a convenient marker of an antitumor immune response in oral squamous cell carcinoma. Our findings suggest that these vessels are a potential immunomodulatory target in this type of cancer. PD-L1 staining in tumor cells correlated with lower T stage, increased infiltration of CD4+ cells, and higher expression of several inflammation-related cytokines. Thus, oral squamous cell carcinomas rich in CD4+ cells may preferentially respond to PD-1/PD-L1 blockade therapy.
\end{abstract}

\section{Introduction}

The majority of oral cancers are squamous cell carcinomas, and their incidence is increasing in many Western countries

Electronic supplementary material The online version of this article (https://doi.org/10.1038/s41379-018-0019-5) contains supplementary material, which is available to authorized users.

$\triangle$ Elin Hadler-Olsen

elin.hadler-olsen@uit.no

1 Department of Medical Biology, Faculty of Health Sciences, University of Troms $\varnothing$ - The Arctic University of Norway, 9037 Troms $\varnothing$, Norway

2 Department of Clinical Pathology, University Hospital of North Norway, 9038 Troms $\varnothing$, Norway
[1]. Oral squamous cell carcinomas are regarded as aggressive cancers, but tumors of the same stage show substantial heterogeneity in progression and response to treatment. Unlike many other cancers, such as breast, lung, and colorectal cancer, there are no reliable biomarkers that predict the aggressiveness or treatment response of an individual oral squamous cell carcinoma [2-4].

The immune system is an important and complex regulator of tumor evolution. Immune suppression promotes tumorigenesis in murine models [5]; and likewise, a number of cancers occur with increased frequency and aggressiveness in immunocompromised patients [6]. Mutated cells express altered proteins or tumor-specific antigens that usually evoke an immune response [7]. However, the intensity and composition of the tumor-associated inflammation vary between patients and may affect prognosis, as demonstrated in melanoma, breast, and ovarian cancer 
Table 1 Primary antibodies for immunohistochemistry

\begin{tabular}{|c|c|c|c|}
\hline Target & Primary antibody & Dilution & Incubation time (min) \\
\hline $\mathrm{T}$ cells & $\begin{array}{l}\text { Rabbit monoclonal anti-CD3, clone 2GV6; Ventana Medical Systems, } \\
\text { Tucson, AZ }\end{array}$ & Pre-diluted & 16 \\
\hline $\mathrm{T}$ helper and $\mathrm{T}$ regulatory cells & Rabbit monoclonal anti-CD4,clone SP35; Ventana Medical Systems & Pre-diluted & 32 \\
\hline Cytotoxic $\mathrm{T}$ cells & Rabbit monoclonal anti CD8, clone SP57; Ventana Medical Systems & Pre-diluted & 32 \\
\hline B cells & Mouse monoclonal anti-CD20, clone L26; Ventana Medical Systems & Pre-diluted & 16 \\
\hline Macrophages & Mouse monoclonal anti-CD68, clone KP-1; Ventana Medical Systems & Pre-diluted & 12 \\
\hline Dendritic cells & $\begin{array}{l}\text { Mouse anti-DC-LAMP/CD208, clone 104G4, \#DDX0190; Dendritics, } \\
\text { Dardilly, France }\end{array}$ & $1: 50$ & 32 \\
\hline PD-L1+ tumor cells & $\begin{array}{l}\text { Rabbit monoclonal anti-PD-L1, clone SP263, \#790-4905; Ventana } \\
\text { Medical Systems }\end{array}$ & Pre-diluted & 16 \\
\hline High-endothelial venules & Rat anti-PNAd, clone MECA-79; Biolegend, San Diego, CA & $1: 25$ & 30 \\
\hline
\end{tabular}

[8-10]. For some cancers, including melanoma and head and neck cancer, an inflamed and a non-inflamed phenotype have been described [11, 12]. Inflamed tumors are characterized by marked T-cell infiltration and high expression of chemokines that can recruit effector $\mathrm{T}$ cells, and are generally associated with a favorable prognosis [13]. Noninflamed tumors, on the other hand, may be linked to defective recruitment of immune cells to the tumor microenvironment, and predict a poor outcome. Lymphocyte extravasation can be regulated by high-endothelial venules, which are blood vessels expressing peripheral node addressin that binds L-selectin on circulating naive lymphocytes [14]. High-endothelial venules are present in secondary lymphoid organs, and develop regularly in chronically inflamed tissue [15]. We have previously reported that the presence of high-endothelial venules is associated with improved survival in oral squamous cell carcinomas [16], which is in line with results from studies on melanoma and breast cancer [17, 18]. However, these vessels are plastic, and their remodeling into regular blood vessels may impair lymphocyte recruitment and precede sentinel lymph node metastasis [19, 20].

Although oral squamous cell carcinomas are mostly of the inflamed phenotype suggesting effective immune surveillance [13], these cancers are generally associated with poor survival. This could be explained by immune editing mechanisms that impair infiltrating lymphocytes and allow tumor progression despite the apparent immune response [21]. Programmed-death ligand 1 (PD-L1)-expressing cells can induce immune suppression by binding the PD-1 receptor (PD-1) on activated $\mathrm{T}$ cells and downregulate effector T-cell functions [22-24]. Reversing immunosuppression through blockade of the PD-1/PD-L1 pathway has shown clinical efficacy in various cancers, including oral squamous cell carcinoma [25-27]. However, studies on the prevalence and prognostic role of PD-L1 expression in oral squamous cell carcinoma are limited, and conflicting results are reported $[22,28,29]$. To date, it remains unclear why only a subset of patients respond to PD-1/PD-L1 blockade treatment.

In a cohort of 75 oral squamous cell carcinoma patients, we recently demonstrated that advanced-stage tumors with high-endothelial venules present were associated with a more pronounced inflammatory response and longer patient survival compared to their high-endothelial venule-negative counterpart [16]. In the present study on the same patient cohort, we explored the hypothesis that high-endothelial venules are master regulators of tumor immunity in oral squamous cell carcinoma by characterizing the immune cell infiltration and cytokine expression in relation to presence of such vessels. Furthermore, we analyzed the association between the tumors' PD-L1 expression and the immune response, and examined a potential relationship between high-endothelial venules and PD-L1 statuses.

\section{Materials and methods}

\section{Patients and material}

In this retrospective study, we included 75 patients diagnosed with primary oral squamous cell carcinoma in the period 1986-2002 from the archives of the Department of Clinical Pathology, University Hospital of North Norway. Follow-ups were continued until January 1, 2012. Patients were included in the study if both clinical data and formalin-fixed, paraffin-embedded tumor specimens (biopsy or surgical resection) were available. Only patients with cancers in the oral cavity proper were included, and they were distributed among the following locations: mobile tongue ( $n=36)$; floor of the mouth $(n=21)$; the alveolar ridge $(n=9)$; buccal mucosa $(n=7)$; palatal arches $(n=1)$ and unspecified oral cavity $(n=1)$. Exclusion criteria were prior radiotherapy to the head and neck area as well as previous oral or oropharyngeal cancer. Sixty-eight percent of the patients received surgery in combination with radiotherapy, 
$15 \%$ concomitant radiotherapy and chemotherapy, $11 \%$ surgery in combination with local neck-dissection, $5 \%$ none or palliative therapy, and $1 \%$ were unknown. We retrieved clinical and histopathologic information from the patients' hospital files, pathology reports, and the Statistics of Norway, Cause of Death Registry, and have earlier published these data in studies analyzing the same patient cohort [16, 30]. Pathological tumor staging was determined according to the most recent (second to fifth) TNM classification from the American Joint Committee on Cancer at the time of diagnosis. There were no changes in the classification of cancers of the lip and oral cavity between the different editions used, so that the description in the fifth edition is relevant for all cases [31]. We conducted the study in line with the REMARK guidelines for tumor marker prognostic studies [32], and following approval from the Regional Committee for Medical and Health Research Ethics, Northern Norway (REK-number 22/2007).

\section{Immunohistochemistry}

We used 4- $\mu$ m-thick sections of formalin-fixed, paraffinembedded tumor specimens from oral squamous cell carcinoma patients for immunohistochemical analyses, and performed both automated and manual staining methods. Specifications of the primary antibodies as well as their incubation conditions are listed in Table 1. We used CD3 as a pan $\mathrm{T}$-cell marker. Various subtypes of $\mathrm{T}$ helper and $\mathrm{T}$ regulatory (Treg) cells express CD4 strongly, whereas monocytes, macrophages, and dendritic cells may show weak expression. Cytotoxic $\mathrm{T}$ cells, and to less extent, natural killer cells and subsets of dendritic cells express CD8. CD20 is a pan B-cell marker, and CD68 is a pan macrophage marker, but it may also stain subsets of lymphocytes, fibroblasts, and endothelial cells. Mature dendritic cells and, to some extent, pneumocytes express DC-lamp/CD208.

\section{Automated staining}

Staining for CD3, CD4, CD8, CD20, CD68, and PD-L1 was done in the automated slide stainer Ventana Benchmark, XT (Ventana, Tucson, AZ, USA) at the Diagnostic Clinic-Clinical Pathology, University Hospital of North Norway, accredited according to the ISO/IEC 15189 standard for the respective staining, as previously published [30]. Briefly, for antigen retrieval, deparaffinized and blocked sections were heat-treated in $0.01 \mathrm{M}$ sodium citrate buffer at $\mathrm{pH}$ 6.0. A cocktail of horseradish peroxidase (HRP)-labeled goat anti-mouse $\mathrm{IgG/IgM}$ and mouse antirabbit secondary antibodies (Ventana UltraView Universal DAB Detection Kit, \#760-500, Roche, Mannheim, Germany) were used for visualization with diaminobenzidine. As these procedures are automatized, the manufacturer controls the incubation time of secondary antibodies. In every run, one slide with known positivity for the different antibodies (tonsil or lymph node) was added as control. Automated staining runs for PD-L1 included in addition a negative control slide with rabbit monoclonal negative control Ig (\#790-4795, Ventana) for each patient. DC-lamp staining was performed in the Ventana Discovery ULTRA autostainer (Ventana). After dewaxing, cell conditioning-1 solution (\#950-124, Ventana) was applied for antigen retrieval for $32 \mathrm{~min}$ at $95^{\circ} \mathrm{C}$. Endogenous peroxidase was blocked by discovery inhibitor CM (\#760-4840, Ventana). After incubation with the DC-lamp primary antibody, the pre-diluted secondary antibody (OmniMap anti-mouse HRP; \#760-4310, Ventana) was loaded for $20 \mathrm{~min}$ followed by HRP amplification and visualization by ChromoMap DAB (\#760-159; Ventana). Counterstaining was performed using the hematoxylin II counterstain reagent (\#790-2208, Ventana). Metastatic lymph node tissue microarray slides of lung cancer were used as positive control. Giemsa staining was performed using BenchMark Special Stains, an automated slide stainer from Ventana. In every run, one slide with known positivity for the stain was added as control.

\section{Manual staining}

Peripheral node addressin staining for the detection of highendothelial venules was performed as previously described [30]. In brief, sections were deparaffinized, rehydrated, subjected to heat-induced antigen retrieval, blocked, and incubated with the peripheral node addressin primary antibody. Next, the sections were incubated with HRP-labeled goat anti-rat light chain secondary antibody (\#AP202P, Millipore, Temecula, CA, diluted 1:250, incubated $30 \mathrm{~min}$ ) and diaminobenzidine (Dako EnVision+ SystemHorseradish Peroxidase, Dako) before being counterstained with Harry's hematoxylin (Sigma-Aldrich, St. Louis, MO). Human lymph node specimens were used as positive control, and specimens with the primary antibody omitted served as negative control.

\section{Immunohistochemical evaluation}

Two trained, independent observers (either EHO and AMW, or SES and IKE) who were blinded to the clinical outcome evaluated the immunohistochemical staining quantitatively and semiquantitatively, as illustrated in the flow chart of Figure S1. We assessed inter-observer variations for all staining except DC-lamp and PD-L1, and the results are listed in Table $\mathrm{S} 1$. Agreement was reached by reevaluation and discussion in case of differing scores. For DC-lamp and PD-L1, the two investigators reached consensus when evaluating the slides together. Micrographs 
were taken with a Leica DFC 420 camera on a Leica DM2000 microscope (Leica, Wetzlar, Germany). Presence of high-endothelial venules was assessed as published earlier [16]. Briefly, we scanned the peripheral node addressin stained tumor-adjacent tissue at low power magnification $(\times 100)$ to identify five areas with high density of highendothelial venules (hotspots). Micrographs of these hotspots were taken at high power magnification $(\times 400)$, and the mean number of high-endothelial venules per section was calculated for each patient by dividing the sum of these vessels in the five hotspots by five. The median number of high-endothelial venules per hotspot for all patients served as cutoff for positive and negative count. Figure S2 shows a micrograph of tumor-associated high-endothelial venules. Giemsa staining was used to identify mast cells and eosinophils as cells with round nuclei and blue/purple granules or cells with lobulated nuclei and bright orange/pink granules, respectively. For each of the cell types, five hotspots in the tumor stroma were identified at low power magnification, and micrographs were taken at $\times 400$ magnification. The total cell number in the hotspots was counted and the mean number calculated. The cutoff for high vs. low count was defined as the median number for the patient cohort, which was 10 for eosinophils and 3 for mast cells. We scored the CD3+, CD4+, CD8+, CD20+, and CD68+ staining semiquantitatively, as it was impossible to apply a quantitative scoring system in densely stained areas. First, we identified the invasive margin of the tumor at low power magnification $(\times 100)$, and micrographs were taken at $\times 400$ magnification, capturing every second visual field of this area. Thus, the number of microscope fields depended on the tumor size. We developed a four-degree scoring scale for the different cell subsets as illustrated in Figure S3, and assigned each micrograph a score: $0=$ no or almost no infiltration; $1=$ mild infiltration; $2=$ moderate infiltration; and $3=$ heavy infiltration. In cases where evaluation was difficult because of weak staining or nonspecific background staining, the positively stained cells were counted, and the scores determined according to the respective cutoff. We calculated a mean score for each section before dichotomizing as low or high if it was $0-1.49$ or $1.5-3$, respectively. PD-L1 expression was assessed based on the percentage of positively labeled tumor cells in each section, and was classified as follows: 0 (labeling in $\leq 5 \%$ of cells), 1 (labeling in $>5 \%$ and $\leq 10 \%$ of cells), 2 (labeling in $>10 \%$ and $\leq 50 \%$ of cells), and 3 (labeling in $>50 \%$ of cells). Expression of DC-lamp was found only in inflamed areas of the tumor stroma and was scored as not expressed (0), slightly expressed [1], moderately expressed [2], or strongly expressed [3] based on semiquantitative evaluation. Both PD-L1 and DC-lamp scores were subsequently grouped into two categories: low expression ( 0 or 1$)$; and high expression (2 or 3 ).

\section{RNA extraction and quality control}

RNA extraction was performed to evaluate gene expression of cytokines. In cases with sufficient residual tumor material, we isolated total RNA from formalin-fixed, paraffin-embedded oral squamous cell carcinoma tissue blocks using the High Pure FFPET RNA Isolation Kit (Roche) following the manufacturer's instructions. In brief, up to four consecutive, 5-10 $\mu \mathrm{m}$-thick sections from each block were deparaffinized and digested with proteinase $\mathrm{K}$, followed by multiple silica-based column purification steps and DNAse I treatment. The RNA on the column was washed several times before being eluted in 20-35 $\mu \mathrm{l}$ elution buffer. We used a mixture of RNA isolated from three different fresh frozen (in liquid nitrogen) human lymphoma specimens as a positive control for further real-time quantitative PCR (qPCR) analyses. The RNeasy Fibrous Tissue mini Kit (Qiagen) was used for RNA isolation according to the manufacturer's protocol. Briefly, the tissue was homogenized using a TissueLyser (Qiagen) before digestion with proteinase $\mathrm{K}$ and centrifugation. The supernatant was washed and treated with DNAse I on a miniature column, and the RNA eluated in $50 \mu 1$ nuclease-free water. We measured total RNA quantity and purity on the NanoDrop spectrophotometer (Thermo Scientific, Wilmington, DE, USA), and assessed RNA integrity using the Experion automated electrophoresis system (Bio-Rad Laboratories, Hercules, USA).

\section{Real-time quantitative PCR}

We used the QuantiTect Reverse Transcription kit (Qiagen) to reverse transcribe 100-200 ng total RNA into cDNA, which was subsequently diluted 1:15 in nuclease-free water. QPCR was performed in duplicates or triplicates using the Light Cycler 96 instrument (Roche). Target cDNA was amplified through 40 cycles in $20 \mu \mathrm{l}$ reactions containing $1 \times$ FastStart Essential DNA Green Master (Roche), $10 \mu$ diluted cDNA (1:15), and $300 \mathrm{nM}$ primers (Table S2). Melting curve analysis was used to verify the specificity of the primers. Controls with the reverse transcriptase omitted and non-template controls were included to test for genomic DNA contamination and carry-over products, respectively. The qPCR amplification efficiency for each gene was calculated from the slope and correlation coefficient (R2) of regression curves from twofold serially diluted cDNA. The $\Delta \Delta \mathrm{Ct}$ method [33] was used to calculate the relative amount of target mRNA normalized against the geometric mean of the reference genes elongation factor 1 alpha, ribosomal protein L27, and ribosomal protein S13. We used geNorm analyses [34] to select three stably expressed reference genes. For association with high-endothelial venules and PD-L1 statuses, we present the results as fold increase compared to the mean of the group with the lowest gene expression ( \pm standard error of mean). 
Table 2 Comparison of low vs. high counts of infiltrating immune cells in OSCC patients ${ }^{\mathrm{a}}$ with and without HEVs using Fisher's exact test

\begin{tabular}{|c|c|c|c|c|c|c|c|c|c|c|}
\hline & \multicolumn{3}{|c|}{ All tumors } & \multirow{3}{*}{$\begin{array}{c}\text { T1/T2 } \\
\text { All HEV+ } \\
\text { No. (\%) }\end{array}$} & \multicolumn{6}{|c|}{$\mathrm{T} 3 / \mathrm{T} 4$} \\
\hline & $\mathrm{HEV}+$ & HEV- & & & \multirow{2}{*}{$\begin{array}{c}\text { All }(\mathrm{HEV}+/-) \\
\text { No. }(\%)\end{array}$} & \multirow[b]{2}{*}{$P$} & \multicolumn{2}{|l|}{$\mathrm{HEV}+$} & HEV- & \multirow[b]{2}{*}{$P$} \\
\hline & No. (\%) & No. $(\%)$ & $P$ & & & & No. (\%) & $P$ & No. (\%) & \\
\hline \multicolumn{11}{|l|}{$\mathrm{CD} 3$} \\
\hline Low & $15(22.4)$ & $6(85.7)$ & 0.002 & $11(21.2)$ & $10(47.6)$ & 0.026 & 4 (28.6) & 0.396 & $6(85.7)$ & 0.002 \\
\hline High & $52(77.6)$ & $1(14.3)$ & & $41(78.8)$ & $11(52.4)$ & & $10(71.4)$ & & $1(14.3)$ & \\
\hline \multicolumn{11}{|l|}{ CD4 } \\
\hline Low & $44(65.7)$ & $5(100.0)$ & 0.170 & $34(65.4)$ & $14(73.7)$ & 0.359 & $9(64.3)$ & 0.587 & $5(100.0)$ & 0.138 \\
\hline High & $23(34.3)$ & $0(0.0)$ & & 18 (34.6) & $5(26.3)$ & & $5(35.7)$ & & $0(0.0)$ & \\
\hline \multicolumn{11}{|l|}{ CD8 } \\
\hline Low & $35(52.2)$ & $5(100.0)$ & 0.061 & $28(53.8)$ & $12(63.2)$ & 0.336 & $7(50.0)$ & 0.517 & $5(100.0)$ & 0.057 \\
\hline High & $32(47.8)$ & $0(0.0)$ & & $24(46.2)$ & $7(36.8)$ & & $7(50.0)$ & & $0(0.0)$ & \\
\hline \multicolumn{11}{|l|}{ CD20 } \\
\hline Low & $26(38.2)$ & $6(85.7)$ & 0.038 & $20(37.7)$ & $12(57.1)$ & 0.104 & $6(42.9)$ & 0.478 & $6(85.7)$ & 0.022 \\
\hline High & $42(61.8)$ & $1(14.3)$ & & $33(62.3)$ & $9(42.9)$ & & $8(57.1)$ & & $1(14.3)$ & \\
\hline \multicolumn{11}{|l|}{ CD68 } \\
\hline Low & $33(48.5)$ & $6(85.7)$ & 0.109 & $22(41.5)$ & $17(81.0)$ & 0.002 & $11(78.6)$ & 0.014 & $6(85.7)$ & 0.034 \\
\hline High & $35(51.5)$ & $1(14.3)$ & & $31(58.5)$ & $4(19.0)$ & & $3(21.4)$ & & $1(14.3)$ & \\
\hline \multicolumn{11}{|l|}{ DC-lamp } \\
\hline Low & $32(51.6)$ & $5(71.4)$ & 0.279 & $25(53.2)$ & $11(52.4)$ & 0.579 & $6(42.9)$ & 0.354 & $5(71.4)$ & 0.314 \\
\hline High & $30(48.4)$ & $2(28.6)$ & & $22(46.8)$ & $10(47.6)$ & & $8(57.1)$ & & $2(28.6)$ & \\
\hline \multicolumn{11}{|c|}{ Eosinophils } \\
\hline Low & $37(55.2)$ & $6(85.7)$ & 0.227 & $26(50.0)$ & $16(76.2)$ & 0.035 & $10(71.4)$ & 0.129 & $6(85.7)$ & 0.082 \\
\hline High & $30(44.8)$ & $1(14.3)$ & & $26(50.0)$ & $5(23.8)$ & & $4(28.6)$ & & $1(14.3)$ & \\
\hline \multicolumn{11}{|c|}{ Mast cells } \\
\hline Low & $32(47.8)$ & $6(85.7)$ & 0.108 & $25(48.1)$ & $13(61.9)$ & 0.209 & $7(50.0)$ & 0.568 & $6(85.7)$ & 0.068 \\
\hline High & $35(52.2)$ & $1(14.3)$ & & 27 (51.9) & $8(38.1)$ & & $7(50.0)$ & & $1(14.3)$ & \\
\hline \multicolumn{11}{|l|}{ PD-L1 } \\
\hline Low & $23(59.0)$ & $4(66.7)$ & 0.544 & $15(50.0)$ & $12(85.7)$ & 0.024 & $8(100.0)$ & 0.010 & $4(66.7)$ & 0.386 \\
\hline High & $16(41.0)$ & $2(33.3)$ & & $15(50.0)$ & $2(14.3)$ & & $0(0.0)$ & & $2(33.3)$ & \\
\hline
\end{tabular}

HEV high-endothelial venule

${ }^{a}$ Analyses for CD3 + cells, eosinophils, and mast cells were carried out in 74 patients, for CD4+ and CD8 + cells in 72 patients, for DC-lamp+ cells in 69 patients, and for PD-L1+ cells in 45 patients. All other analyses included 75 patients

For survival and correlation analyses, we dichotomized the results in low and high expression based on the median of fold increase as cutoff.

\section{Statistical analysis}

We used SPSS software version 22.0 for Windows (IBM, Armonk, NY) and Microsoft Excel 2013 (Microsoft, Redmond, WA) for all calculations. Inter-observer variability for the various quantitative and semiquantitative cell counts was analyzed using the Spearman correlation test, and correlation between two variables was assessed by the Fisher's exact test. We used univariate Kaplan-Meier analyses to calculate disease-specific death rates and plot disease-specific survival curves, and the log-rank test to evaluate the statistical significance. Multivariate analyses were done using a stepwise forward multiple Cox regression model. Linear regression analyses of standard curves derived from serially diluted cDNA were used to estimate qPCR amplification efficiency. The significance level was set at $P<0.05$, and the borderline significance level at $P<0.09$.

\section{Results}

\section{High-endothelial venules predict T- and B-cell infiltration into oral squamous cell carcinomas}

We recently demonstrated a correlation between the presence and morphology of high-endothelial venules and the 
A

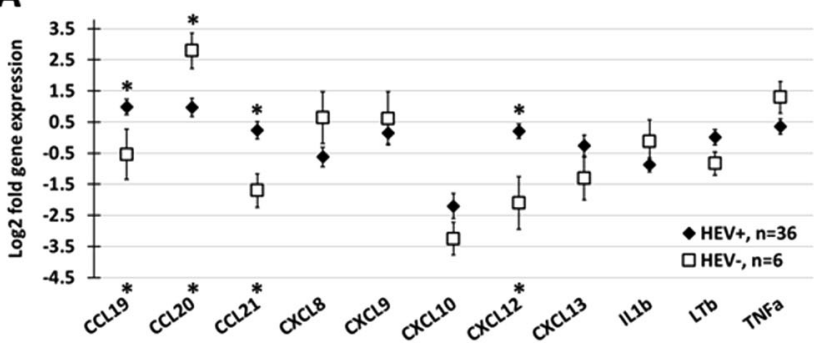

C

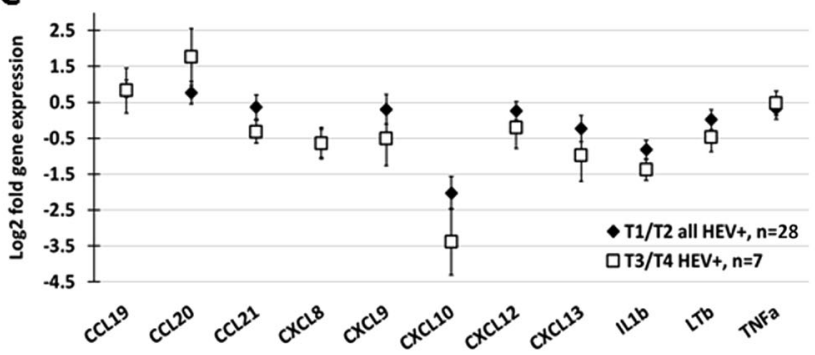

B

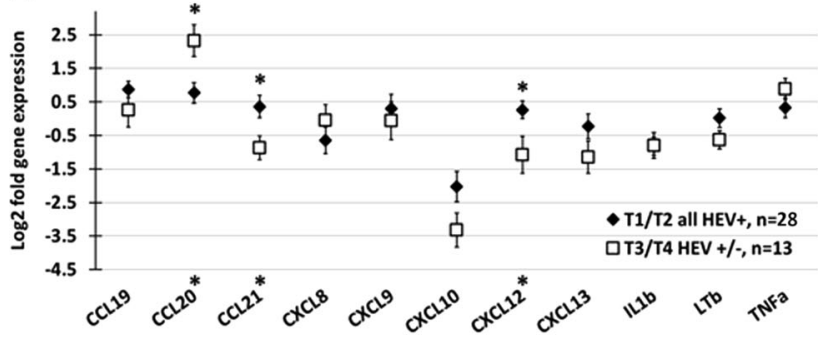

D

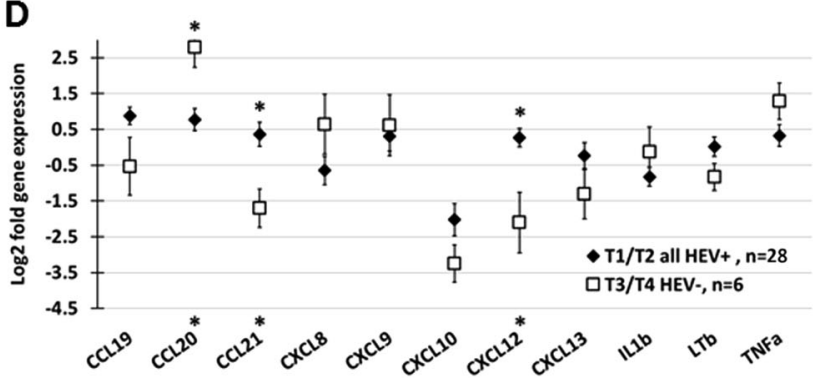

Fig. 1 Log2-fold gene expression of various chemokines in formalin-fixed paraffin-embedded oral squamous cell carcinoma tissue samples. Comparison of the following groups: a high-endothelial venule (HEV)-positive vs. HEV-negative tumors; b T1/T2 vs. T3/T4 tumors; c T1/T2 vs. T3/T4 tumors with HEVs; and d T1/T2 vs. T3/T4 tumors without HEVs. All T1/T2 tumors were HEV-positive. Error bars indicate \pm standard error of the mean, and $* P<0.05$. The $P$-value was calculated using two sample $T$-test

intensity of the inflammatory infiltrate in oral squamous cell carcinomas [16]. Now, to determine the composition of the immune infiltrate, we immunohistochemically stained tumor sections from 75 oral squamous cell carcinoma patients with antibodies against the following immune cell markers: CD3 (pan T cell); CD4 (T helper, Treg cell); CD8 (cytotoxic T cell); CD20 (pan B cell); CD68 (pan macrophage), and DC-lamp/CD208 (mature dendritic cell). We also performed Giemsa staining to identify eosinophils and mast cells. The high-endothelial venule-negative tumors were generally less infiltrated with immune cells compared to the high-endothelial venule-positive tumors (Table 2). The differences were statistically significant for CD3+ $(P$ $=0.002)$ and $\mathrm{CD} 20+(P=0.038)$ cells, and borderline significant for $\mathrm{CD} 8+$ cells $(P=0.061)$.

To determine cytokines associated with high-endothelial venules, we conducted qPCR analysis of 42 of the 75 oral squamous cell carcinoma tissue samples, of whom 36 were high-endothelial venule-positive and 6 were negative (Fig. 1a). The lymphoid chemokines CCL19, CCL21, and CXCL12 were significantly higher expressed in the highendothelial venule-positive compared to the tumors without these vessels $(P=0.04,0.02$, and 0.001 , respectively). The inflammatory chemokine CCL20, however, was significantly lower expressed in the high-endothelial venule-positive than in the -negative tumors $(P=0.02)$. Taken together, our results shows that high-endothelial venule-positive tumors are more heavily infiltrated with lymphocytes than tumors without such vessels, which may be promoted by the chemokines CCL19, CCL21, and CXCL12.

\section{Oral squamous cell carcinomas with high- endothelial venules retain an inflamed phenotype even at high $\mathrm{T}$ stage}

In the same oral squamous cell carcinoma patient cohort, we have earlier found that high-endothelial venules were present in all T1/T2 tumors, compared to two-thirds of the T3/ T4 tumors [16], and hypothesized that absence of these vessels in advanced tumor stages may suppress the immune reaction. To elaborate this theory, we compared the immune cell infiltrate and cytokine expression in the T1/T2 tumors with the T3/T4 tumors, as well as the T3/T4 tumors subdivided into high-endothelial venule-positive and -negative. We found a significantly higher amount of infiltrating CD3 $+\mathrm{T}$ cells, CD68+ macrophages, and eosinophils in the T1/ $\mathrm{T} 2$ tumors (all high-endothelial venule-positive) compared to the T3/T4 tumors (high-endothelial venule-positive and -negative) as presented in Table $2(P=0.026,0.002$, and 0.035 , respectively). Dividing the T3/T4 tumors into highendothelial venule-positive and -negative revealed that the positive had an immune cell infiltrate that resembled the T1/ T2 tumors, except for fewer CD68+ cells in the T3/T4 tumors $(P=0.014)$. In contrast, the high-endothelial venule-negative T3/T4 tumors showed lower scores than T1/T2 tumors for all immune cell subsets analyzed, except $\mathrm{CD} 4+$ cells and DC-lamp + dendritic cells that were not significantly different.

QPCR analysis of $28 \mathrm{~T} 1 / \mathrm{T} 2$ tumor samples (all highendothelial venule-positive) and $13 \mathrm{~T} 3 / \mathrm{T} 4$ tumor samples (high-endothelial venule-positive, $n=7$; high-endothelial 


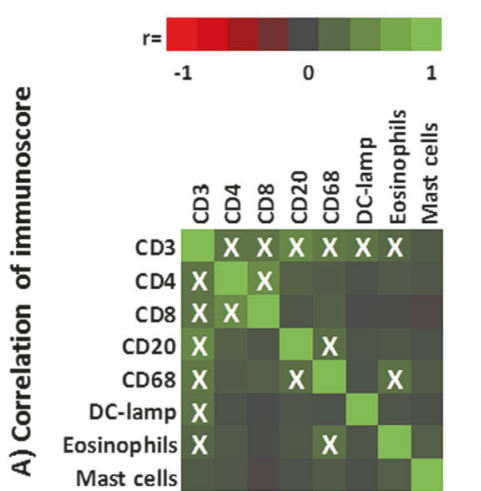

Fig. 2 Heatmaps of Pearson correlation coefficients. The heatmaps show results from bivariate correlation analyses of the following parameters in the oral squamous cell carcinomas: a immune cell immunohistochemical scores; b gene expression pattern; and $\mathbf{c}$ gene
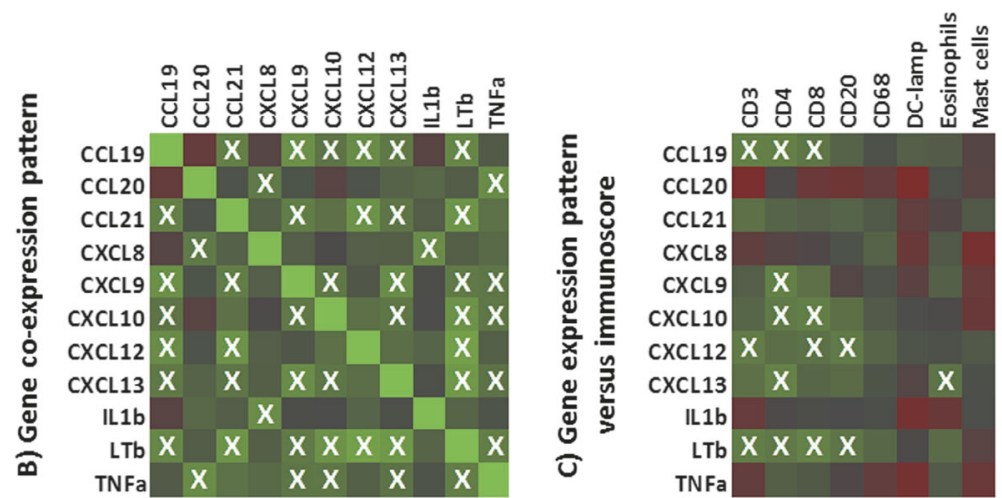

expression pattern and immune cell immunohistochemical scores. White crosses in the heatmaps show significant correlation between the different variables with $P<0.05$

venule-negative, $n=6$ ) revealed higher levels of CCL21 and CXCL12, and lower levels of CCL20 in the T1/T2 compared to the T3/T4 tumors $(P=0.04,0.02$, and 0.01 , respectively; Fig. 1b). In accordance with the results from immune cell analyses, the high-endothelial venule-positive T3/T4 tumors displayed no statistically significant or borderline significant differences to the T1/T2 tumors' cytokine expression (Fig. 1c). The high-endothelial venule-negative T3/T4 tumors, however, had significantly lower expression of CCL21 and CXCL12 and higher expression of CCL20 $(P=0.02,0.04$, and 0.01 , respectively; Fig. 1d) than the T1/T2 tumors. Together, our results suggest that the presence of high-endothelial venules supports an inflamed phenotype even in large (T3/T4) tumors.

\section{T-cell infiltration predicts an immune cell-rich tumor microenvironment and is associated with CCL19, CXCL12, and lymphotoxin b expression}

Cytokines, chemokines, and cells of the innate and adaptive immune system interact in complex attraction, activation, and inhibition networks. To reveal putative regulatory mechanisms in the oral squamous cell carcinoma immune infiltrate, we performed correlation analyses between the expression level of cytokines, the various immune cell scores, and the clinicopathological variables. The immunohistochemical score for CD3 $+\mathrm{T}$ cells showed statistically significant, positive correlations with all other immune cells analyzed except for mast cells (Fig. 2a). There were few significant correlations between the other immune cell subsets. With the exception of CXCL8, CCL20, and interleukin-1 $\beta$ (IL1b), we found numerous positive, significant correlations between the expression levels of the different cytokines analyzed (Fig. 2b). Lymphocyte score (CD3+, CD4+, CD8+, and CD20+ cells) showed significant positive correlation with one or several of the cytokines
CCL19, CXCL9, CXCL10, CXCL12, CXCL13, and lymphotoxin b (LTb; Fig. 2c). None of the cytokines was significantly correlated with the number of CD68+ macrophages, DC-lamp + dendritic cells, or mast cells, suggesting that these cells are regulated by mechanisms distinct from the other studied immune cell populations. Correlation data of the patients' clinicopathological variables with immune cell scores and cytokine expression levels are presented in Table S3. Of note, the group size for many of the variables is small, smoking history and alcohol consumption are patient-reported data, and treatment choice is strongly influenced by tumor stage and comorbidity. Altogether, our results show that high numbers of CD3+ $\mathrm{T}$ cells in oral squamous cell carcinoma are correlated with increased infiltration of a number of other immune cells, and suggest that the cytokines CCL19, CXCL12, and LTb may have a prominent role in promoting immune cell infiltration through their association with $\mathrm{T}$ cells.

\section{PD-L1 expression in oral squamous cell carcinoma cells correlates with increased infiltration of CD4+ cells and small tumor size}

Cancer cells may escape immune surveillance by expressing PD-L1, which induces apoptosis in PD-1 expressing $\mathrm{T}$ lymphocytes, most notably cytotoxic $\mathrm{T}$ cells. PD-1/PD-L1 checkpoint blockade has shown promising results in various types of cancer, but only subgroups of patients respond to the treatment. To increase the understanding of PD-L1 expression and its association with the immune microenvironment, we analyzed PD-L1 expression in 45 tumors, randomly chosen from the 75 oral squamous cell carcinoma patient cohort, and determined correlation to infiltrating immune cells, cytokine expression, and the presence of high-endothelial venules. In all, 18 (40\%) of the 45 tumor sections stained positive for PD-L1. The staining was either 
Table 3 Comparison of low vs. high numbers of infiltrating immune cells in oral squamous cell carcinoma patients ${ }^{\mathrm{a}}$ with low and high PDL1 scoring using Fisher's exact test

\begin{tabular}{|c|c|c|c|}
\hline & $\begin{array}{l}\text { PD-L1 low } \\
\text { No. }(\%)\end{array}$ & $\begin{array}{l}\text { PD-L1 high } \\
\text { No. }(\%)\end{array}$ & $P$ \\
\hline \multicolumn{4}{|l|}{ CD3 } \\
\hline Low & $10(37.0)$ & $4(22.2)$ & \multirow[t]{2}{*}{0.237} \\
\hline High & $17(63.0)$ & $14(77.8)$ & \\
\hline \multicolumn{4}{|l|}{ CD4 } \\
\hline Low & $21(80.8)$ & $8(47.1)$ & \multirow[t]{2}{*}{0.025} \\
\hline High & $5(19.2)$ & $9(52.9)$ & \\
\hline \multicolumn{4}{|l|}{ CD8 } \\
\hline Low & $18(69.2)$ & $7(41.2)$ & \multirow[t]{2}{*}{0.066} \\
\hline High & $8(30.8)$ & $10(58.8)$ & \\
\hline \multicolumn{4}{|l|}{$\mathrm{CD} 20$} \\
\hline Low & $11(40.7)$ & $9(50.0)$ & \multirow[t]{2}{*}{0.379} \\
\hline High & $16(59.3)$ & $9(50.0)$ & \\
\hline \multicolumn{4}{|l|}{ CD68 } \\
\hline Low & $15(55.6)$ & $9(50.0)$ & \multirow[t]{2}{*}{0.475} \\
\hline High & $12(44.4)$ & $9(50.0)$ & \\
\hline \multicolumn{4}{|l|}{ DC-lamp } \\
\hline Low & $14(58.3)$ & $9(50.0)$ & \multirow[t]{2}{*}{0.411} \\
\hline High & $10(41.7)$ & $9(50.0)$ & \\
\hline \multicolumn{4}{|c|}{ Eosinophils } \\
\hline Low & $17(63.0)$ & $8(44.4)$ & \multirow[t]{2}{*}{0.179} \\
\hline High & $10(37.0)$ & $10(55.6)$ & \\
\hline \multicolumn{4}{|c|}{ Mast cells } \\
\hline Low & $13(48.1)$ & $8(44.4)$ & \multirow[t]{2}{*}{0.525} \\
\hline High & $14(51.9)$ & $10(55.6)$ & \\
\hline
\end{tabular}

${ }^{a}$ Analyses for CD4+ and CD8+ cells were carried out in 43 patients and for DC-lamp + cells in 42 patients. All other analyses included 45 patients

membranous and/or cytoplasmic as demonstrated in Figure S4. We did not assess PD-L1 positivity of stromal cells because of diffuse staining. Tumors with high PD-L1 expression showed increased infiltration of CD4+ and CD8 + cells compared to those with low PD-L1 expression $(P=$ 0.025 and 0.066 , respectively; Table 3 ). They also expressed higher levels of a number of cytokines, including CCL19, CCL21, CXCL9, CXCL10, CXCL13, and LTb ( $P$ $=0.006, P=0.010, P<0.001, P<0.001, P<0.001$, and $P=0.011$, respectively; Fig. 3). PD-L1 score was not correlated with patients' survival $(P=0.207$; Table S4). The T3/T4 tumors had significantly lower PD-L1 immune score than the T1/T2 tumors $(P=0.024$; Table 2$)$. Dividing the T3/T4 tumors into high-endothelial venule-positive and -negative revealed that all positive T3/T4 tumors had low PD-L1 expression, whereas the negative showed no statistically significant difference in PD-L1 expression compared to the T1/T2 tumors $(P=0.010$ and 0.386 , respectively). Our results demonstrate that PD-L1 expression in tumor

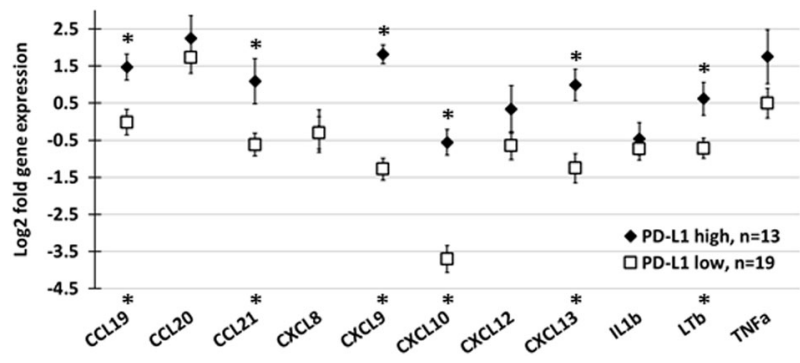

Fig. 3 Comparison of $\log 2$-fold gene expression of various chemokines and cytokines in formalin-fixed paraffin-embedded oral squamous cell carcinoma tissue samples with high and low programmeddeath ligand 1 (PD-L1) score. Error bars indicate \pm standard error of the mean, and $* P<0.05$. The $P$-value was calculated using two sample $T$-test

cells is associated with a tumor microenvironment rich in $\mathrm{CD} 4+$ cells and inflammatory cytokines, and that highendothelial venules are negatively correlated with PD-L1 in large tumors (T3/T4).

\section{High-endothelial venules are more powerful prognosticators than the $\mathrm{N}$ stage and separate components of the oral squamous cell carcinoma immune infiltrate}

Many components of the immune infiltrate have been suggested as potential prognosticators for oral cancer, but study results are contradictory. Univariate Kaplan-Meier survival analyses of the immune cell scores and cytokine expression levels in our oral squamous cell carcinoma cohort showed that high numbers of $\mathrm{CD} 20+\mathrm{B}$ cells and CD68+ macrophages were significant prognosticators for longer 5-year survival ( $P=0.002$ and 0.027 , respectively; Fig. 4 and Table S4). We have previously reported that the high-endothelial venule status, the tumor site as well as the $\mathrm{T}$ and $\mathrm{N}$ stages were significant prognostic factors in the same patient cohort [16]. All variables that were significantly associated with disease-specific death in univariate analyses were entered into multivariate Cox regression analyses. The proportional hazards assumption was fulfilled for all variables (Figure S5 and [16]). Only the T stage $(P<0.001)$ and the high-endothelial venule status $(P=0.002)$ were independent predictors for diseasespecific death (Table 4). These results suggest that highendothelial venules are more important prognostic factors than the $\mathrm{N}$ stage and the subsets of the immune infiltrate assessed in this study.

\section{Discussion}

In this study, we show that the presence of highendothelial venules is indicative of a favorable immune 


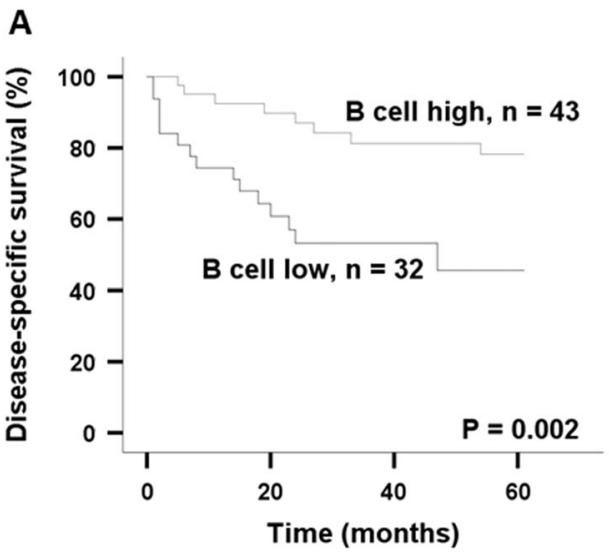

Fig. 4 Kaplan-Meier analysis of 5-year disease-specific survival for patients with oral squamous cell carcinoma. High counts of a CD20+ $\mathrm{B}$ cells and b CD68+ macrophages were associated with improved survival $(P=0.002$ and 0.027 , respectively). The Kaplan-Meier
B

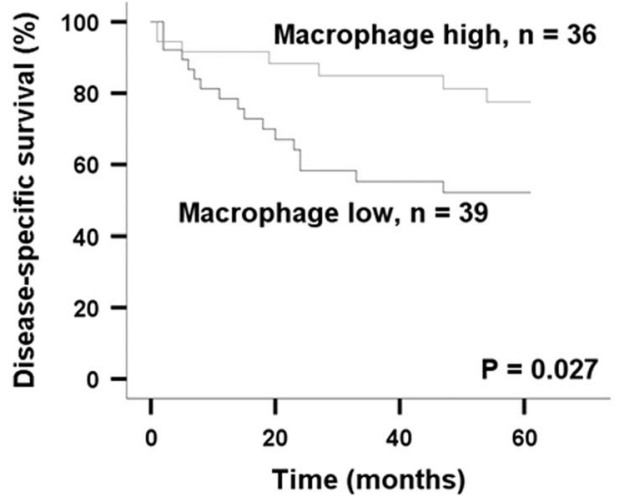

curves show a 5-year disease-specific survival rate of $81.4 \%$ for CD20 high vs. $50 \%$ for CD20 low tumors (a), and $80.6 \%$ for CD68 high vs. $56.4 \%$ for CD68 low tumors (b). The $P$-value was calculated using the log-rank test

Table 4 Multivariate analysis of 5-year disease-specific death according to Cox's proportional hazards model ${ }^{\mathrm{a}}$

\begin{tabular}{llll}
\hline Variable & Hazard ratio & $95 \%$ CI & $P$ \\
\hline T stage (T1/T2 $(n=49)$ vs. T3/T4 $(n=20))$ & 0.121 & $0.045-0.321$ & $<0.001$ \\
HEV (positive $(n=63)$ vs. negative $(n=6))$ & 0.147 & $0.044-0.490$ & 0.002 \\
\hline
\end{tabular}

${ }^{a}$ Only 69 patients were analyzed because the cases with the unknown $\mathrm{T}$ and $\mathrm{N}$ stages were taken out from the calculations

microenvironment in oral squamous cell carcinoma, and that these vessels seem to counter-act immunosuppressive mechanisms and improve patient survival irrespective of the tumors' $T$ stage. PD-L1-expressing tumor cells correlated positively to a tumor microenvironment rich in CD4+ cells, but had no prognostic significance. Our results suggest that high-endothelial venules have a pivotal role in shaping an antitumor immune response in oral squamous cell carcinoma, and that PD-1/PD-L1-targeted immunotherapy might be specifically successful in patients with tumors rich in CD4+ cells. To the best of our knowledge, this is the first study to investigate jointly the role of PD-L1 and highendothelial venules in oral squamous cell carcinoma, two components of the immune infiltrate with important regulatory functions.

High density of tumor-associated high-endothelial venules has earlier been associated with improved survival and inflammation in several cancers, including melanoma, oral, and breast cancers $[16,17,35]$, and in the present study, we aimed to determine the composition of the highendothelial venule associated immune infiltrate in oral squamous cell carcinoma. We found that the highendothelial venule-positive tumors were heavily infiltrated by $\mathrm{CD} 3+\mathrm{T}$ cells, $\mathrm{CD} 8+$ cytotoxic $\mathrm{T}$ cells, and CD20+ B cells, which is in accordance with previous findings in melanoma and breast cancer $[17,18]$. Infiltration of CD8+ cytotoxic $\mathrm{T}$ cells, and especially a high $\mathrm{CD} 8+/$ Treg ratio have been associated with a favorable prognosis in a variety of human solid tumors including ovarian, cervical and oral cancer [36-38]. However, of the immune cell subsets analyzed in our study, only increased infiltration of CD20+ B cells and CD68 + macrophages were significantly correlated with improved patient outcome in univariate analyses. The presence of B cells has earlier been associated with positive outcome in head and neck cancer patients $[39,40]$. B cells mediate a humoral immune response by producing tumorspecific antibodies, and these cells often localize and cooperate with $\mathrm{T}$ cells to facilitate potent, long-term antitumor responses [41]. In contrast to our results, previous studies have mainly found a negative association between macrophages and patient survival in oral cancer [42-44]. The conflicting results may be due to differences in immunohistochemical procedures and scoring. Besides, tumor-associated macrophages appear in different functional states that may vary between tumors and within specific tumor areas [45, 46], and the pan macrophage marker CD68 does not distinguish between these phenotypes. The CD68 antibody, as well as several of the other antibodies used in this study, is not strictly specific for a single cell type. Accurate assessment of immune cell subsets requires multiple markers for each subtype, which is laborious and costly to incorporate in a day-to-day routine clinical practice. In the present study, only the highendothelial venule- and T-statuses were independent 
positive prognosticators for 5-year disease-specific survival. This indicates that these vessels are more relevant as prognostic markers than other components of the tumor microenvironment analyzed, and strengthens their role as a potent surrogate marker of an effective antitumor immune response. Immunohistochemical detection of highendothelial venules is simple and reliable [16], and implementation in clinical pathology practice could thus be straightforward.

Chemokines in and around high-endothelial venules are thought to be crucial for lymphocyte extravasation into lymphoid organs [47]. In the present study, the gene expression of the lymphoid chemokines CXCL12, CCL19, and CCL21 was upregulated in the high-endothelial venulepositive tumors. Expression of CXCL12 is an important attractant for naive $\mathrm{T}$ and $\mathrm{B}$ cells [48, 49], and has a potential role in high-endothelial venule-mediated $\mathrm{T}$-cell trafficking into lymph nodes in vitro [50]. CCL19 and CCL21 interact with CCR7 on lymphocytes, and trigger efficient T-cell homing. Thus, increased expression of CCL19, CCL21, and CXCL12 in high-endothelial venulepositive tumors supports an important role for these vessels in homing of naive $\mathrm{T}$ cells into oral squamous cell carcinoma. Besides their role in lymphocyte homing, CCL19 and CCL21 stimulate migration and maturation of dendritic cells [51]. Through LT expression, dendritic cells promote maintenance of a mature high-endothelial venule phenotype [52]. Therefore, downregulation of CCL21 in T3/T4 tumors may be indicative of a tumor microenvironment with less infiltrating mature dendritic cells, causing dedifferentiation of high-endothelial venules into normal blood vessels [19, 20]. However, we did not find a significant association between DC-lamp, a marker for mature dendritic cells, and high-endothelial venule score. To further study the association between dendritic cells and these vessels, we also performed high-endothelial venule/dendritic cell double staining, but faced technical difficulties (data not shown). In future studies, assessing markers for tumor angiogenesis could be a valuable supplement to investigate high-endothelial venule plasticity, and to shed light on development and maintenance of these vessels in advanced tumors. Furthermore, validating the results of the present study in a larger patient cohort with a higher number of high-endothelial venule-negative tumors is important, as the low number of these tumors in the present study may skew the statistical results.

High-endothelial venule-negative tumors expressed higher levels of the chemokine CCL20 than -positive tumors. CCL20 attracts cells expressing the CCR6 receptor, such as dendritic cells and memory and effector $\mathrm{T}$ cells. Several studies have found that CCL20 is a chief attractant of Treg cells, a distinct lineage of CD4+ T cells that suppresses antitumor immune responses [53-55]. Interestingly, tumor cells may also express CCR6, and accordingly, CCL20 has been associated with migration and metastases of cancer cells [56]. We did not specifically stain for Treg cells nor CCR6, but speculate that the downregulation of CCL20 in high-endothelial venule-positive oral squamous cell carcinomas could help sustain an antitumor immune response by avoiding recruitment of Treg cells. This would be interesting to investigate in future studies. In summary, we show that both the early and the advanced highendothelial venule-positive tumors displayed higher levels of tumor-suppressive components of the immune infiltrate than the negative. Along with the vessels' independent prediction of improved survival, this suggests that highendothelial venules promote a tumor-suppressive immune response irrespective of the tumors' $\mathrm{T}$ stage.

Tumor progression often correlates with immune evasion. In several cancers, including oral squamous cell carcinoma, immune suppression may be facilitated by PD-L1 that inhibits T-cell functions [22, 23]. In the present study, tumors with high PD-L1 score showed a significant increase in CD $4+$ cell infiltration $(P=0.025)$. Increased numbers of immunosuppressive CD4+ Treg cells, as well as dysfunctional $\mathrm{T}$ lymphocytes have previously been found to predict immunosuppressive properties in oral cancer patients [5759]. We also found a borderline significant association between PD-L1+ tumor cells and infiltrating CD8+ cells $(P=0.066)$, as well as the expression level of a number of inflammation-related cytokines. This suggests that PD-L1 expression by itself does not lead to a non-inflamed tumor environment, but may influence the efficacy of the immune reaction. This is in accordance with several other studies that report PD-L1 expression to be associated with cytotoxic $\mathrm{T}$ cells that can induce PD-L1 expression in an interferon (IFN)- $\gamma$ dependent manner [60-62]. CXCL9 is an IFN- $\gamma$ inducible chemokine, and we found a perfect linear relationship between PD-L1 score and CXCL9 expression ( $r=$ 1; data not shown). Human papilloma virus (HPV)/p16positive tumors had significantly lower CXCL9 expression than HPV/p16-negative tumors. A correlation between HPV-positivity and PD-L1 expression has previously been reported in oropharyngeal cancer [63]. Due to the low number of HPV/p16-positive cancers in our study, the correlation between the virus, CXCL9, and PD-L1 expression in oral cancer needs validation in a study with a higher amount of HPV-positive patients.

Tumors in the floor of the mouth and tumors of cigarette smokers showed significantly lower PD-L1 expression than tumors at other subsites in the oral cavity and tumors of patients who were non-smokers. In the same patient cohort, we have previously reported a correlation between alcohol and tobacco consumption and tumors in the floor of the mouth [64]. Furthermore, tumors in the floor of the mouth expressed less CCL21 than tumors in the other anatomical 
sites (Table S5), and tumors with low PD-L1 expression expressed lower levels of CCL21 than tumors with strong PD-L1 expression (Fig. 3). Whether there is a direct effect of CCL21 and/or tobacco on PD-L1 expression would be interesting to investigate in future studies. Interestingly, high PD-L1 expression was also correlated to a lower T stage $(P=0.037)$, and we found a significantly lower score of PD-L1-expressing tumor cells in the high-endothelial venule-positive T3/T4 compared to the T1/T2 tumors $(P=$ $0.010)$. This may suggest that high-endothelial venulepositive large tumors have overcome PD-L1-mediated immunosuppression, which could contribute to the increased survival of patients with high-endothelial venulepositive T3/T4 tumors. PD-1/PD-L1 blockade is a new immunotherapeutic approach in the combat against cancer, and the clinical success of this treatment correlates to some extent with the tumors' PD-L1 expression [25-27]. It can be speculated that PD-L1-expressing T1/T2 tumors and highendothelial venule-negative T3/T4 tumors with marked Tcell infiltration are good candidates for PD-1/PD-L1 checkpoint-targeting therapies.

In conclusion, we show that high-endothelial venules are markers of a favorable antitumor immune microenvironment in oral squamous cell carcinoma, and stronger prognosticators than other subsets of the immune infiltrate. As detection of these vessels is easy and reliable, highendothelial venule status may serve as a valuable supplement to stratify oral squamous cell carcinoma patients for targeted therapeutic approaches. Oral squamous cell carcinomas are generally immunosuppressive tumors with poor patient outcome. Understanding the mechanisms that drive immune cell recruitment and generate effective antitumor responses may provide opportunities to develop new immunomodulatory targets and thereby increase the consistently low survival rates of patients with oral cancer.

Acknowledgements The study was supported by grants from The North Norwegian Health Authorities. The authors thank the technical staff at the Department of Clinical Pathology, University Hospital of North Norway, Bente Mortensen, Mehrdad Rakaee Khanehkenari, and Kjersti Julin at the Department of Medical Biology, University of Troms $\varnothing$ - The Arctic University of Norway (UiT) for excellent technical help. We are also grateful for statistical advice from Professor Tom Wilsgård at the Department of Community Medicine, UiT.

\section{Compliance with ethical standards}

Conflict of interest The authors declare that they have no conflict of interest.

\section{References}

1. Annertz K, Anderson H, Palmer K, et al. The increase in incidence of cancer of the tongue in the Nordic countries continues into the twenty-first century. Acta Otolaryngol. 2012;132:552-7.
2. Soland TM, Brusevold IJ. Prognostic molecular markers in cancer —quo vadis? Histopathology. 2013;63:297-308.

3. Holdenrieder S. Biomarkers along the continuum of care in lung cancer. Scand J Clin Lab Invest Suppl. 2016;245:S40-5.

4. Al-Hajeili M, Shields AF, Hwang JJ, et al. Molecular testing to optimize and personalize decision making in the management of colorectal cancer. Oncology (Williston Park). 2017;31:301-12.

5. Swann JB, Smyth MJ. Immune surveillance of tumors. J Clin Invest. 2007;117:1137-46.

6. Krisl JC, Doan VP. Chemotherapy and transplantation: the role of immunosuppression in malignancy and a review of antineoplastic agents in solid organ transplant recipients. Am J Transplant. 2017;17:1974-91.

7. Marcus A, Gowen BG, Thompson TW, et al. Recognition of tumors by the innate immune system and natural killer cells. Adv Immunol. 2014;122:91-128.

8. Marrogi AJ, Munshi A, Merogi AJ, et al. Study of tumor infiltrating lymphocytes and transforming growth factor-beta as prognostic factors in breast carcinoma. Int $\mathrm{J}$ Cancer. 1997;74:492-501.

9. Zhang L, Conejo-Garcia JR, Katsaros D, et al. Intratumoral $\mathrm{T}$ cells, recurrence, and survival in epithelial ovarian cancer. $\mathrm{N}$ Engl J Med. 2003;348:203-13.

10. Clemente CG, Mihm MC Jr, Bufalino R, et al. Prognostic value of tumor infiltrating lymphocytes in the vertical growth phase of primary cutaneous melanoma. Cancer. 1996;77:1303-10.

11. Gajewski TF. The next hurdle in cancer immunotherapy: overcoming the non-T-cell-inflamed tumor microenvironment. Semin Oncol. 2015;42:663-71.

12. Woo SR, Corrales L, Gajewski TF. The STING pathway and the $\mathrm{T}$ cell-inflamed tumor microenvironment. Trends Immunol. 2015;36:250-6.

13. Mandal R, Senbabaoglu Y, Desrichard A, et al. The head and neck cancer immune landscape and its immunotherapeutic implications. JCI Insight. 2016;1:e89829.

14. van Zante A, Rosen SD. Sulphated endothelial ligands for Lselectin in lymphocyte homing and inflammation. Biochem Soc Trans. 2003;31:313-7.

15. Hayasaka H, Taniguchi K, Fukai S, et al. Neogenesis and development of the high endothelial venules that mediate lymphocyte trafficking. Cancer Sci. 2010;101:2302-8.

16. Wirsing AM, Rikardsen OG, Steigen SE, et al. Presence of tumour high-endothelial venules is an independent positive prognostic factor and stratifies patients with advanced-stage oral squamous cell carcinoma. Tumour Biol. 2016;37:2449-59.

17. Martinet L, Garrido I, Girard JP. Tumor high endothelial venules (HEVs) predict lymphocyte infiltration and favorable prognosis in breast cancer. Oncoimmunology. 2012;1:789-90.

18. Martinet L, Le Guellec S, Filleron T, et al. High endothelial venules (HEVs) in human melanoma lesions: major gateways for tumor-infiltrating lymphocytes. Oncoimmunology. 2012;1: 829-39.

19. Lee SY, Chao-Nan Q, Seng OA, et al. Changes in specialized blood vessels in lymph nodes and their role in cancer metastasis. J Transl Med. 2012;10:206.

20. Qian CN, Berghuis B, Tsarfaty G, et al. Preparing the "soil": the primary tumor induces vasculature reorganization in the sentinel lymph node before the arrival of metastatic cancer cells. Cancer Res. 2006;66:10365-76.

21. Tong CC, Kao J, Sikora AG. Recognizing and reversing the immunosuppressive tumor microenvironment of head and neck cancer. Immunol Res. 2012;54:266-74.

22. Zandberg DP, Strome SE. The role of the PD-L1:PD-1 pathway in squamous cell carcinoma of the head and neck. Oral Oncol. 2014;50:627-32. 
23. Lyford-Pike S, Peng S, Young GD, et al. Evidence for a role of the PD-1:PD-L1 pathway in immune resistance of HPVassociated head and neck squamous cell carcinoma. Cancer Res. 2013;73:1733-41.

24. Keir ME, Butte MJ, Freeman GJ, et al. PD-1 and its ligands in tolerance and immunity. Annu Rev Immunol. 2008;26:677-704.

25. Brahmer JR, Tykodi SS, Chow LQ, et al. Safety and activity of anti-PD-L1 antibody in patients with advanced cancer. N Engl J Med. 2012;366:2455-65.

26. Topalian SL, Hodi FS, Brahmer JR, et al. Safety, activity, and immune correlates of anti-PD-1 antibody in cancer. N Engl J Med. 2012;366:2443-54.

27. Ibrahim R, Stewart R, Shalabi A. PD-L1 blockade for cancer treatment: MEDI4736. Semin Oncol. 2015;42:474-83.

28. Hsu MC, Hsiao JR, Chang KC, et al. Increase of programmed death-1-expressing intratumoral CD8 $\mathrm{T}$ cells predicts a poor prognosis for nasopharyngeal carcinoma. Mod Pathol. 2010;23:1393-403.

29. Badoual C, Hans S, Merillon N, et al. PD-1-expressing tumorinfiltrating $\mathrm{T}$ cells are a favorable prognostic biomarker in HPVassociated head and neck cancer. Cancer Res. 2013;73:128-38.

30. Wirsing AM, Rikardsen OG, Steigen SE, et al. Characterisation and prognostic value of tertiary lymphoid structures in oral squamous cell carcinoma. BMC Clin Pathol. 2014;14:38.

31. American Joint Committee on Cancer. Staging of cancer at specific anatomic sites, lip and oral cavity. In: Fleming ID, Cooper JS, Henson DE, et al.,editors. AJCC cancer staging manual. 5th ed. Philadelphia: Lippincott-Raven Publishers; 1997. p. 24-30.

32. Altman DG, McShane LM, Sauerbrei W, et al. Reporting recommendations for tumor marker prognostic studies (REMARK): explanation and elaboration. BMC Med. 2012;10:51.

33. Livak KJ, Schmittgen TD. Analysis of relative gene expression data using real-time quantitative PCR and the 2(-Delta Delta C(T)) Method. Methods. 2001;25:402-8.

34. Pattyn F, Speleman F, De Paepe A, et al. RTPrimerDB: the realtime PCR primer and probe database. Nucleic Acids Res. 2003;31:122-3.

35. Martinet L, Garrido I, Filleron T, et al. Human solid tumors contain high endothelial venules: association with $\mathrm{T}$ - and $\mathrm{B}$ lymphocyte infiltration and favorable prognosis in breast cancer. Cancer Res. 2011;71:5678-87.

36. Sato E, Olson $\mathrm{SH}$, Ahn J, et al. Intraepithelial CD8+ tumorinfiltrating lymphocytes and a high $\mathrm{CD} 8+$ /regulatory $\mathrm{T}$ cell ratio are associated with favorable prognosis in ovarian cancer. Proc Natl Acad Sci USA. 2005;102:18538-43.

37. Jordanova ES, Gorter A, Ayachi O, et al. Human leukocyte antigen class I, MHC class I chain-related molecule A, and CD8 +/regulatory T-cell ratio: which variable determines survival of cervical cancer patients? Clin Cancer Res. 2008;14:2028-35.

38. Watanabe Y, Katou F, Ohtani H, et al. Tumor-infiltrating lymphocytes, particularly the balance between $\mathrm{CD} 8(+) \mathrm{T}$ cells and CCR4 $(+)$ regulatory $\mathrm{T}$ cells, affect the survival of patients with oral squamous cell carcinoma. Oral Surg Oral Med Oral Pathol Oral Radiol Endod. 2010;109:744-52.

39. van Herpen CM, van der Voort R, van der Laak JA, et al. Intratumoral rhIL-12 administration in head and neck squamous cell carcinoma patients induces $\mathrm{B}$ cell activation. Int $\mathrm{J}$ Cancer. 2008;123:2354-61.

40. Pretscher D, Distel LV, Grabenbauer GG, et al. Distribution of immune cells in head and neck cancer: CD8+ T-cells and CD20+ B-cells in metastatic lymph nodes are associated with favourable outcome in patients with oro- and hypopharyngeal carcinoma. BMC Cancer. 2009;9:292.

41. Nelson BH. CD20+ B cells: the other tumor-infiltrating lymphocytes. J Immunol. 2010;185:4977-82.
42. Fujii N, Shomori K, Shiomi T, et al. Cancer-associated fibroblasts and CD163-positive macrophages in oral squamous cell carcinoma: their clinicopathological and prognostic significance. J Oral Pathol Med. 2012;41:444-51.

43. Marcus B, Arenberg D, Lee J, et al. Prognostic factors in oral cavity and oropharyngeal squamous cell carcinoma. Cancer. 2004;101:2779-87.

44. Ni YH, Ding L, Huang XF, et al. Microlocalization of CD68+ tumor-associated macrophages in tumor stroma correlated with poor clinical outcomes in oral squamous cell carcinoma patients. Tumour Biol. 2015;36:5291-8.

45. Mantovani A, Sozzani S, Locati M, et al. Macrophage polarization: tumor-associated macrophages as a paradigm for polarized M2 mononuclear phagocytes. Trends Immunol. 2002;23:549-55.

46. Lewis CE, Pollard JW. Distinct role of macrophages in different tumor microenvironments. Cancer Res. 2006;66:605-12.

47. Miyasaka M, Tanaka T. Lymphocyte trafficking across high endothelial venules: dogmas and enigmas. Nat Rev Immunol. 2004;4:360-70.

48. Pablos JL, Amara A, Bouloc A, et al. Stromal-cell derived factor is expressed by dendritic cells and endothelium in human skin. Am J Pathol. 1999;155:1577-86.

49. Campbell JJ, Hedrick J, Zlotnik A, et al. Chemokines and the arrest of lymphocytes rolling under flow conditions. Science. 1998;279:381-4.

50. Phillips R, Ager A. Activation of pertussis toxin-sensitive CXCL12 (SDF-1) receptors mediates transendothelial migration of T lymphocytes across lymph node high endothelial cells. Eur J Immunol. 2002;32:837-47.

51. Marsland BJ, Battig P, Bauer M, et al. CCL19 and CCL21 induce a potent proinflammatory differentiation program in licensed dendritic cells. Immunity. 2005;22:493-505.

52. Moussion C, Girard JP. Dendritic cells control lymphocyte entry to lymph nodes through high endothelial venules. Nature. 2011;479:542-6.

53. Liu JY, Li F, Wang LP, et al. CTL- vs Treg lymphocyte-attracting chemokines, CCL4 and CCL20, are strong reciprocal predictive markers for survival of patients with oesophageal squamous cell carcinoma. Br J Cancer. 2015;113:747-55.

54. Lee JJ, Kao KC, Chiu YL, et al. Enrichment of human CCR6+ regulatory $\mathrm{T}$ cells with superior suppressive activity in oral cancer. J Immunol. 2017;199:467-76.

55. Chen KJ, Lin SZ, Zhou L, et al. Selective recruitment of regulatory $\mathrm{T}$ cell through CCR6-CCL20 in hepatocellular carcinoma fosters tumor progression and predicts poor prognosis. PLoS ONE. 2011;6:e24671.

56. Frick VO, Rubie C, Keilholz U, et al. Chemokine/chemokine receptor pair CCL20/CCR6 in human colorectal malignancy: an overview. World J Gastroenterol. 2016;22:833-41.

57. Reichert TE, Strauss L, Wagner EM, et al. Signaling abnormalities, apoptosis, and reduced proliferation of circulating and tumor-infiltrating lymphocytes in patients with oral carcinoma. Clin Cancer Res. 2002;8:3137-45.

58. Hoffmann TK, Dworacki G, Tsukihiro T, et al. Spontaneous apoptosis of circulating $\mathrm{T}$ lymphocytes in patients with head and neck cancer and its clinical importance. Clin Cancer Res. $2002 ; 8: 2553-62$

59. Gasparoto TH, de Souza Malaspina TS, Benevides L, et al. Patients with oral squamous cell carcinoma are characterized by increased frequency of suppressive regulatory $\mathrm{T}$ cells in the blood and tumor microenvironment. Cancer Immunol Immunother. 2010;59:819-28.

60. Spranger S, Spaapen RM, Zha Y, et al. Up-regulation of PD-L1, IDO, and $\mathrm{T}$ (regs) in the melanoma tumor microenvironment is driven by CD8(+) T cells. Sci Transl Med. 2013;5:200ra116.

61. Iwai Y, Okazaki T, Nishimura H, et al. Microanatomical localization of PD-1 in human tonsils. Immunol Lett. 2002;83:215-20. 
62. Shi F, Shi M, Zeng Z, et al. PD-1 and PD-L1 upregulation promotes CD8(+) T-cell apoptosis and postoperative recurrence in hepatocellular carcinoma patients. Int J Cancer. 2011;128:887-96.

63. Hong AM, Vilain RE, Romanes S, et al. PD-L1 expression in tonsillar cancer is associated with human papillomavirus positivity and improved survival: implications for anti-PD1 clinical trials. Oncotarget. 2016;7:77010-20.
64. Rikardsen OG, Bjerkli IH, Uhlin-Hansen L, et al. Clinicopathological characteristics of oral squamous cell carcinoma in Northern Norway: a retrospective study. BMC Oral Health. 2014; $14: 103$. 